\title{
LA VIGENCIA DE UN MÉTODO EN ARQUEOLOGÍA: Sobre la propuesta de Periodificación Cultural del Área Andina de John H. Rowe
}

\author{
Carlos Del Águila Chávez*
}

\begin{abstract}
Resumen
La idea original del presente ensayo ha estado esperando desde el año 2006 para que pueda ser publicado por el MNAAHP, el cual dirigí por esos años, junto con otro grupo de ensayos dedicados a evaluar la obra teórico-metodológica de Rowe. Este proyecto editorial que lo llamamos en esa oportunidad "Homenaje a John Rowe", no ha podido ver la luz, a pesar de los esfuerzos de los directivos del Museo de Pueblo Libre. Sin embargo, debo reconocer el valioso e inteligente desprendimiento de su actual director Christian Mesía para que, por las ediciones sanmarquinas, podamos publicar algunos de estos materiales. Que mejor reconocimiento entonces para Craig Morris que publicar estos ensayos que abordan la secuencia cultural de Chincha y reconsidera las fortalezas de un método que, al propio decir de Craig: Se resiste a morir.
\end{abstract}

Palabras clave

Cronologías, secuencias, Chincha, Costa Sur, periodos.

\begin{abstract}
The original idea of the this paper has been waiting from the year 2006 in order that it could be published by the MNAAHP, which I directed these years, together with another group of articles dedicated to evaluating Rowe's theoretical - methodological work. This publishing project, that we call it in that opportunity "Honoring to John Rowe ", could not be realized in spite of the efforts of the executives of the Pueblo Libre Museum. Nevertheless, I must recognize the valuable and intelligent detachment of his current director Christian Mesía in order that, for the sanmarquinas editions, we could publish some of these materials. The best recognition for Craig Morris is to publish these articules that approach the cultural sequence of Chincha and reconsiders the strengths of a method that, to the own one to say of Craig: It refuses to die.
\end{abstract}

\section{Keywords}

Chronologies, sequences, Chincha, South Coast, periods.

* Arqueólogo. Director General del Centro Cultural de San Marcos.

Correo electrónico: crdela@hotmail.com 


\section{INTRODUCCIÓN}

Desarrollar un ensayo sobre John Howland Rowe, sobre su obra, sobre su aporte a la arqueología de los Andes o sobre el significado de su contribución en la periodificación del Antiguo Perú, es una labor que debe implicar una serie de análisis que paso a desarrollar. Estos análisis deben involucrar, incluso, aspectos como las que debió tener Rowe al esbozar y/o organizar los datos de su investigación; aspectos como por ejemplo, bosquejar notas a mano o a máquina, cruzar información algo dispersa o articulada de ciertos datos o, simplemente, desarrollar una rigurosa metodología de investigación que le permitió estructurar, en líneas generales, la propuesta cronológica más aceptada aún vigente para los Andes Centrales del Antiguo Perú.

Me propongo indagar sobre estos aspectos, específicamente para el caso de la Costa Sur -que resultó ser el globo de ensayo- precisamente porque amerita, a la luz de los nuevos datos, establecer precisiones y valorar aciertos del esquema que Rowe estableció hace ya cuarenta y cinco años y que todavía, a mi parecer y salvando los esquemas, funciona para el territorio andino.

\section{REMEMBRANZA}

Antes que nada, me permito resaltar, en este homenaje al recientemente desaparecido peruanista; tal vez en la única oportunidad que pude hablar con el Dr. Rowe no estuvo planeado, pero resultó para mi ser uno de esos momentos en la vida de un arqueólogo que se impregna en la mente y queda grabado de por vida. Sería más o menos el verano de 1990, cuando con Javier Alcalde y Leonid Velarde realizábamos trabajos de gabinete en la casa de nuestro maestro y jefe Luis Lumbreras, casi todas las tardes de ese año realizamos el estudio del material cerámico de prospección del valle de Chincha recuperados por el Proyecto Arqueológico-Histórico Chincha-Pisco que el Instituto Andino de Estudios Arqueológicos (INDEA) y el American Museum of Natural History de New York (AMNH) realizaron entre los años 1984 y 1985; éramos estudiantes $y$, creo que fue uno de los viernes de ese verano, que nuestra colega y amiga Marcela Ríos de Lumbreras nos avisó por la tarde que el Dr. Rowe llegaba a Lima junto con Patricia Lyon y que avisaba a todos los amigos que podía a una reunión con él. La posibilidad de escuchar a John Rowe y Patricia Lyon que estaban de paso por pocas horas en Lima y querían conversar con amigos y colegas era una oportunidad que no se podía dejar pasar. Me pareció notable la idea que John $\mathrm{H}$. Rowe, a quien todo estudiante universitario peruano que se respete en el Perú ha leído, esté solicitando conversar con colegas peruanos e intercambiar ideas.

Nos sentimos afortunados, casi privilegiados, esa tarde fue especial, esperamos mucho, se fumaron muchos cigarros y se tomaron muchos cafés. Así que llegó; la sala principal de la casa del maestro Lumbreras rebasaba de gente, habremos sido unos 25 o 30 convocados. Lo noté nervioso, entusiasmado pero nervioso ... y comenzó, nos habló horas de unas ideas que estaba procesando sobre la percepción que tenía de la ciudadela de Machu Picchu y su uso especial con el entorno, con su paisaje asociado, con la recurrencia de las formas de las montañas que se divisaban de ciertos lugares estratégicos del sitio Inca.

Lejos de entender completamente lo que nos decía, que evidentemente eran ideas, bocetos de ideas, lo que Luis Lumbreras nos orientó a valorar de lo que escuchábamos era su capacidad aún presente pese a su avanzada edad, de estructurar organizadamente muchos datos e ir dándoles forma de hipótesis de trabajo. Casi abrumados y con mucha timidez, algunos de nosotros, al finalizar su disertación, hicimos preguntas sobre temas específicos y muy puntuales; Lumbreras ahondó más en cuestionamientos teóricos y se inició la tertulia más fascinante que haya podido presenciar hasta ahora; muy ligera, fresca, descartando y elaborando hipótesis de trabajo como si se recitaran poemas. Finalmente no sé si continuó desarrollando las ideas que nos presentó esa noche o si la descartó; solo me queda el recuerdo de la fascinación de ver a 
un hombre de avanzada edad, utilizando su brillantez intelectual como si fuera un adolescente. Nunca olvidaré eso, tampoco su humildad y pasión al contarnos sus cosas, sus ideas. Con este recuerdo, quiero empezar este ensayo el cual intentará en todo momento, ejercitar los aspectos que se consiguen plasmar solo en esas ocasiones, cuando se aprende a observar, a ver más allá, cuando se aprende a contar historia; muchos maestros intentan enseñarte eso, muy pocos lo consiguen.... A pesar que tan solo lo vimos esa vez, el Dr. Rowe lo consiguió con nosotros.

\section{EL MÉTODO}

Rowe se hizo cargo en 1957 de uno de los proyectos arqueológicos más ambiciosos de su tiempo realizados en el Perú. La Comisión Fullbright de Intercambio Educativo financió a un grupo de arqueólogos norteamericanos liderados por él para ejecutar estudios en toda la costa peruana, el centro de sus operaciones las concentró en el departamento de Ica, por ser esta región la que mayor información acumulada y colecciones formalizadas tenía el país. Junto con Dorothy Menzel y Laurence Dawson, constituyeron el programa científico que permitió esbozar el panorama cronológico del mundo andino. Las consecuencias de estos experimentos arqueológicos todavía se encuentran en permanente debate en nuestros tiempos.

El fundamento metodológico lo presentó en la revista Arqueología y Sociedad № 6 de la UNMSM de 1971 y los argumentos teóricos de base ya los había alcanzado en diversas publicaciones años atrás (ver Rowe 1959a). En ellos, explicita con detalle, paso a paso cómo llega a establecer una organización coherente de ciertos rasgos de las colecciones, basado en la experiencia desarrollada en la arqueología clásica europea y la clasificación de aspectos estilísticos a partir de los denominados "extremos conocidos".

Este principio parte del supuesto de una cadena de continuidad de cambios de ciertos rasgos, los cuales tienen referentes asociativos inmediatos con lo cronológicamente conocido en el tiempo histórico por un lado y con lo totalmente desconocido por el otro. Así, y soportado con la información contextual y estratigráfica, se estructura la columna referencial de la ubicación de los materiales en el tiempo.

El argumento central del método es muy simple: asumiendo esta continuidad cultural de los rasgos estilísticos, como una ley universal del arte de todos los tiempos, podemos organizar las características artísticas desde lo más simple a lo más complejo o desde la imperfección a la perfección de la tecnología o desde la expresión directa de un mensaje iconográfico hasta la expresión abstracta e indirecta del mismo. Es decir, la organización de cierto tipo de materiales va a obtener los mismos resultados cronológicos asumiendo este principio; es mas, fortaleciendo esta organización de materiales con la información contextual, vamos a tener una herramienta de organización cronológica lo suficientemente eficaz para reconstruir procesos a lo largo de diferentes periodos de tiempo; como un inicio o punto de partida y no como un examen concluyente.

La prueba del método asociativo estilístico de Rowe, como lo define Lumbreras, la desarrolló en el Sur Andino, por varios motivos, pero sobretodo porque se constituía en la región más intensamente investigada y lamentablemente huaqueada de la región andina; de modo que, las colecciones formadas de esas zonas eran -y lo son aún- abundantes. Efectivamente, el punto de partida de Rowe fueron las colecciones locales y la de los museos centrales; así, fue armando la línea cronológica de tiempo con los materiales enteros representativos, ubicando en el extremo conocido más antiguo a los materiales chavinoides y en el extremo conocido más reciente a los materiales Inca. Luego, "al lado y para adelante" de los materiales chavinoides, lo que se parecían a estos, de modo que ubicó las colecciones Paracas Cavernas, seguidas de las Necrópolis. Por el otro extremo, ubicó "al lado pero para atrás" a los materiales parecidos a los Inca, o sea los de Ica y Chincha; incluso, los menos parecidos a los Inca pero todavía parecidos 
a los Ica/Chincha, es decir, los Epigonales, fueron ubicados "al lado y para atrás" de los Ica/Chincha. Finalmente, los que no se parecían a nada, es decir los Nazca, fueron ubicados al centro de la línea cronológica. Este fue el ejercicio de punto de partida del método (Ver Esquema 1).

Luego vinieron, los complementos informativos de cada uno de estos estilos y la información de estratigrafía que confirmaba ciertos antecedentes o consecuentes. Además, se agregó la información más antigua a la chavinoide que se venía identificando en la costa central con los trabajos de Fréderic Engel y finalmente los trabajos del periodo Precerámico o Lítico a cargo de Edward P. Lanning. Así se armó la primera columna cronológica metodológicamente organizada que permitiría, posteriormente, corroborar la información o descartarla; el tiempo y las investigaciones posteriores, en líneas generales las han corroborado más que descartado.

Considero vigente esta propuesta por varios motivos, sin que ello signifique que no tenga errores o imperfecciones; y es que para esas épocas, la seriación como procedimiento metodológico venía siendo aplicada cada vez con más fuerza en los Andes, la cual, fusionada con el método asociativo estilístico que promovía Rowe, permitió que las estructuras cronológicas de "referencia" sean muy precisas o por lo menos muy cautelosas. Eran tiempos de los innumerables nombres de estilos que día a día se identificaban en los diferentes valles; aún así, la construcción de esta columna fue el comienzo de un orden referencial que todavía mantene- mos. Esto es un aporte, creo yo, aún vigente.

En los cuadros que presentó Menzel en Arqueología y Sociedad № 6, se apreciaba ese panorama, los transcribimos porque amerita reconocer el esfuerzo de construcción que está plasmado en ellos y en los cuales tuvo mucha participación Rowe (Ver Cuadro 1). Estos en comparación con los cuadros que el Proyecto Chincha maneja para el valle (Ver Cuadro 2), permiten comprobar, a la luz de datos de excavación y de interpretación más recientes que las estructuras base de la línea cronológica funcionan bien, las imperfecciones se detectan, obviamente, en las precisiones de cada momento o época, ya que, para algunos casos, la convivencia de estilos es algo lógicamente habitual y que en aquellos tiempos era poco tomado en cuenta.

En estos cuadros es notable cómo ya se van identificando, luego de la organización de los materiales, procesos externos a la región de Ica que manifiestan una sólida presencia e influencia en los valles vecinos. De esta forma, al comparar el cuadro de Menzel y Rowe con el preparado por Lumbreras para el PAHCHP, podemos verificar que, las estructuras generales de la columna cronológica no han cambiado. Debo aclarar acá que el cuadro preparado por Lumbreras se sustenta no solamente en la información bibliográfica del momento sino también en sistemáticas excavaciones arqueológicas donde se ha podido evaluar y comprobar "estratigráficamente" la superposición de unos elementos culturales con otros. Inclusive, durante toda la segunda mitad de los noventa

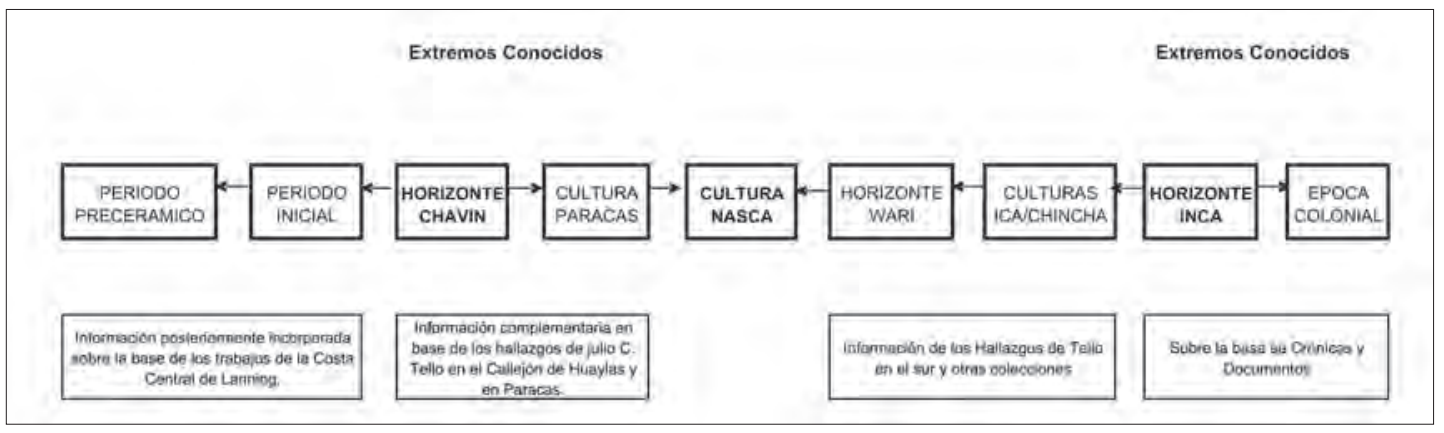

Esquema 1 


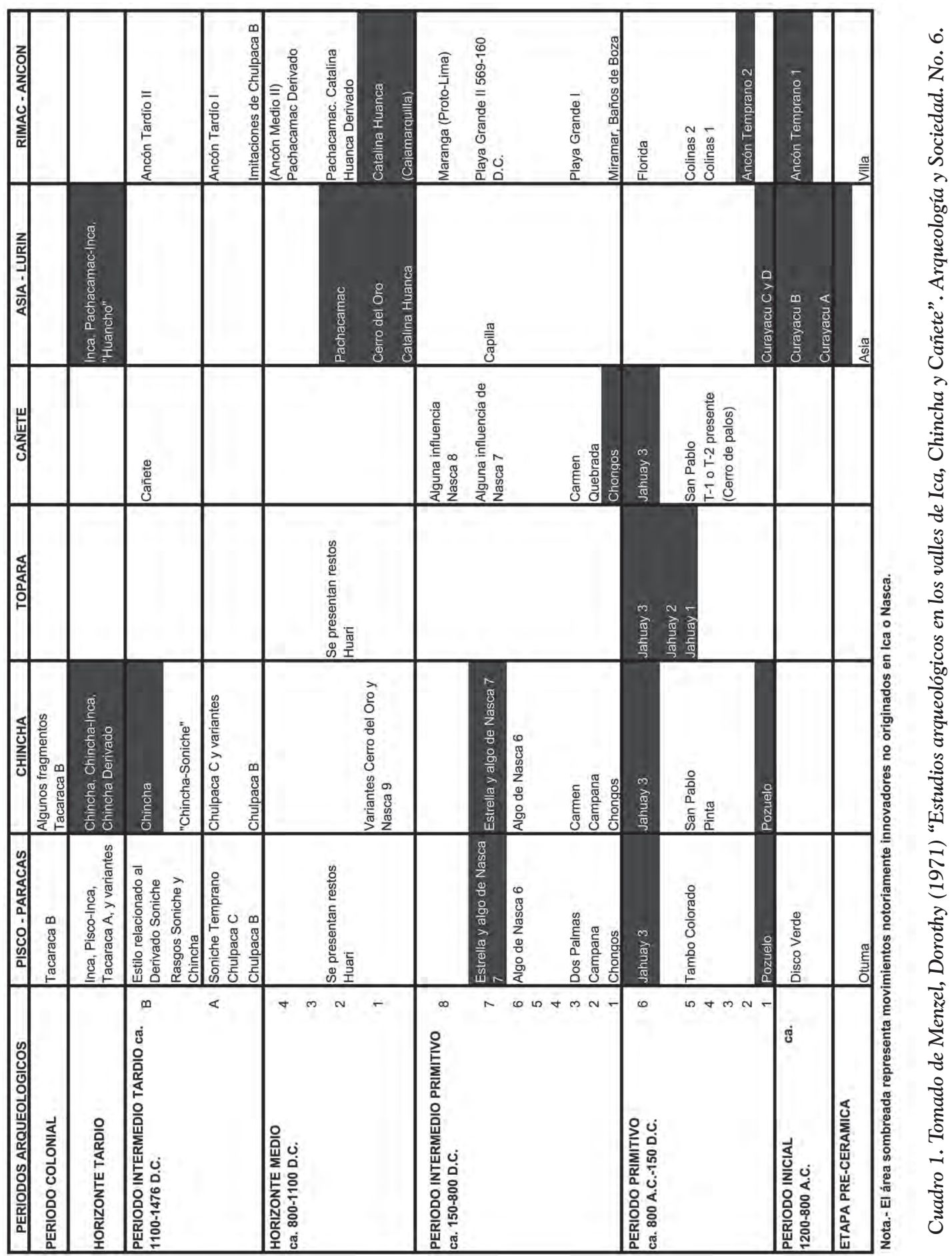




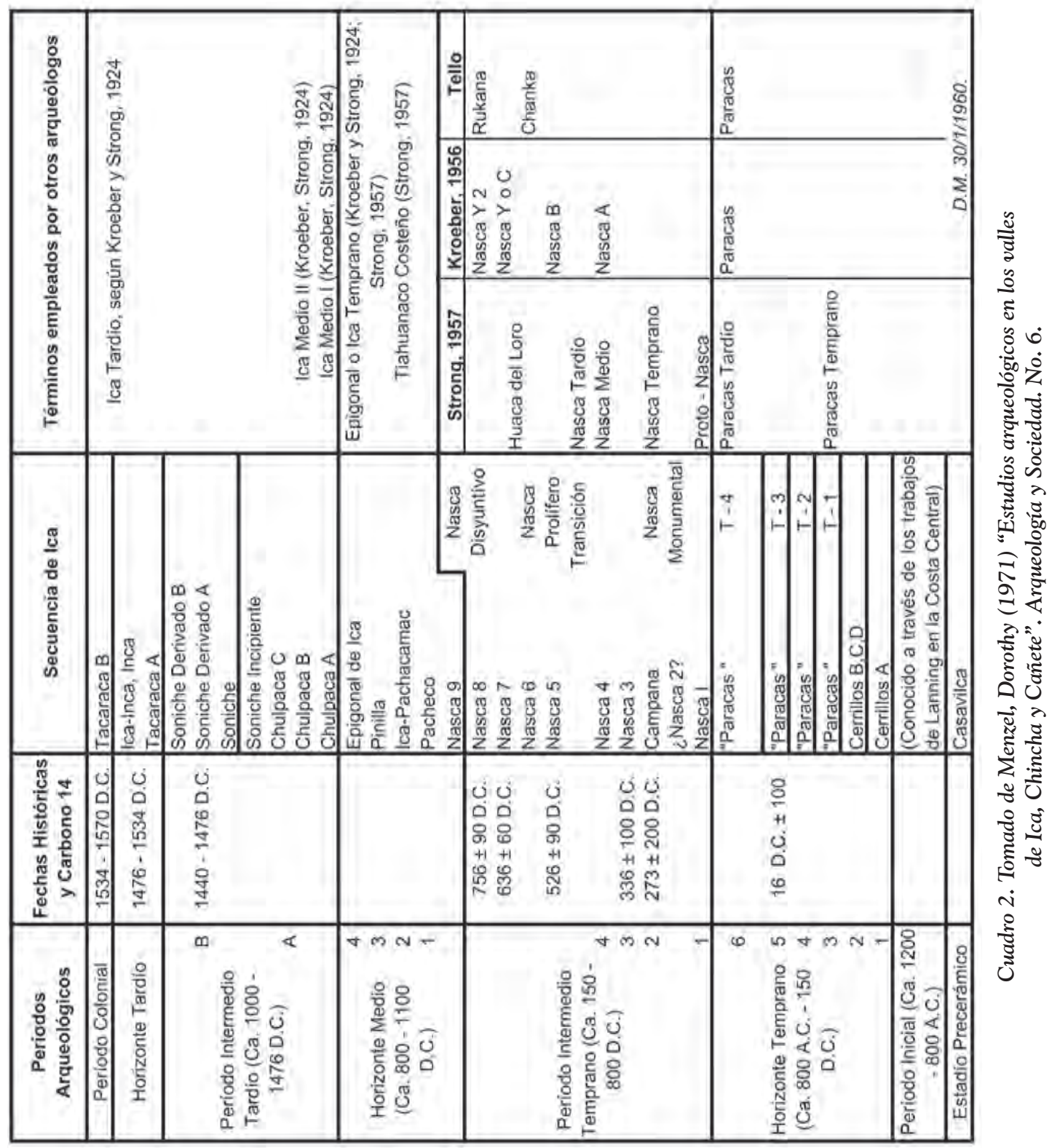


y entre el 2000 y 2005, el autor y Javier Alcalde se hicieron cargo de nuevas intervenciones arqueológicas en el valle que, lejos de aportar en datos nuevos, ha permitido reconfirmar, en líneas generales, la vigencia de la organización "base" que Rowe y Menzel propusieran hacia finales de los cincuenta.

\section{SeCuencias COMPARADAS}

La secuencia del sur del Perú es, en resumidas cuentas, el modelo de la secuencia específica del valle de Chincha. Sin muchas diferencias, salvo la denominación de algunos estilos locales, los periodos cronológicos reflejan los mismos patrones de proceso cultural y esto es un modelo que, con algunas precisiones más o menos, sigue funcionando.

Haciendo un recuento comparativo de los dos esquemas que analizo en este artículo, vale decir, la planteada por el equipo de Rowe en 1971 y la planteada por el equipo de Lumbreras en 1984, podemos comprobar la vigencia metodológica y las posibles líneas futuras de investigación que perfeccionen la secuencia y la hagan más sólida; veamos:

En la primera época denominada Horizonte Temprano, para el valle de Chincha se identificaban los estilos Pinta, Jahuay III, Chongos y Campana, las cuales corresponden, en el caso de Pinta a la época Cavernas de Paracas y los tres siguientes estilos a la época Necrópolis de Paracas, esto se ha corroborado con los estudios de Wallace en los años 1957 y 1959 y las verificaciones del Proyecto Chincha entre los años 1985 y 2005; excavaciones realizadas por Velarde en el sitio de Pampa de los Gentiles (2000-01) y las realizadas por Lumbreras Flores (2000) en el sitio de Pozuelos, reconfirman esta secuencialidad de las épocas Cavernas y Necrópolis.

El Periodo Intermedio Temprano, definido en la secuencia maestra presentada por Menzel como resultado de los trabajos realizados por Wallace, propone dos estilos cerámicos que de alguna manera ya habían sido identificados por
Uhle como Proto Chincha; estos son los estilos Carmen (con 2 fases) y Estrella (con 4 fases), estos estilos, sólidamente caracterizados y secuencialmente correlacionados, lo que permite identificarlos, asociados o por separados. Estos estilos sólo se manifiestan en el valle de Chincha, por lo menos así lo vemos en los sitios que hemos estudiado. En definitiva hay una fuerte relación con los las fases 2-3 y 7 de la Secuencia de Nasca con los estilos Carmen y Estrella de la secuencia de Chincha respectivamente, pero conservan una autonomía de estilo que funciona con cierta coherencia en los momentos de ocupación.

Lo notable del aporte de las investigaciones recientes del Proyecto Chincha es la presencia Wari, que en la década de los 50, era muy limitada pero presente. Con los hallazgos reportados en el Boletín de la PUCP sobre Wari en el año 2003, se presenta el caso a UPIS San José, donde se registra y rescata de manera fortuita una especie de ofrenda de vasijas consistentemente Wari de las primeras épocas, asociado a material Estrella, lo cual se verifica en las excavaciones del Proyecto Chincha en La Cantera; además de la presencia más sólida del estilo Huacarones identificado por Carmichael en el año 1995, el cual se asocia a las épocas finales del denominado Horizonte Medio. Futuros estudios de estos materiales permitirán fortalecer esta problemática.

El Intermedio Tardío ha sido el periodo más evaluado tanto por Menzel como por las campañas de campo tanto en La Centinela, Tambo de Mora cerca de la línea de playa como en Pampa de La Pelota, valle adentro en años recientes. Está por demás comprobado la definición de dos momentos cronológicos de este periodo: uno de presencia netamente Chincha y otro con convivencia Chincha-Inka. Las excavaciones de varias temporadas en Tambo de Mora y las realizadas en Pampa de la Pelota corroboran estos datos con bastante certeza. Menzel profundizó en notables estudios publicados en Ñawpa Pacha y Arqueología y Sociedad, la complejidad estilística de este periodo; el Proyecto Chincha contribuyó en este aspecto a correlacionar ciertos estilos dentro de dos grandes momentos, tratándose de 


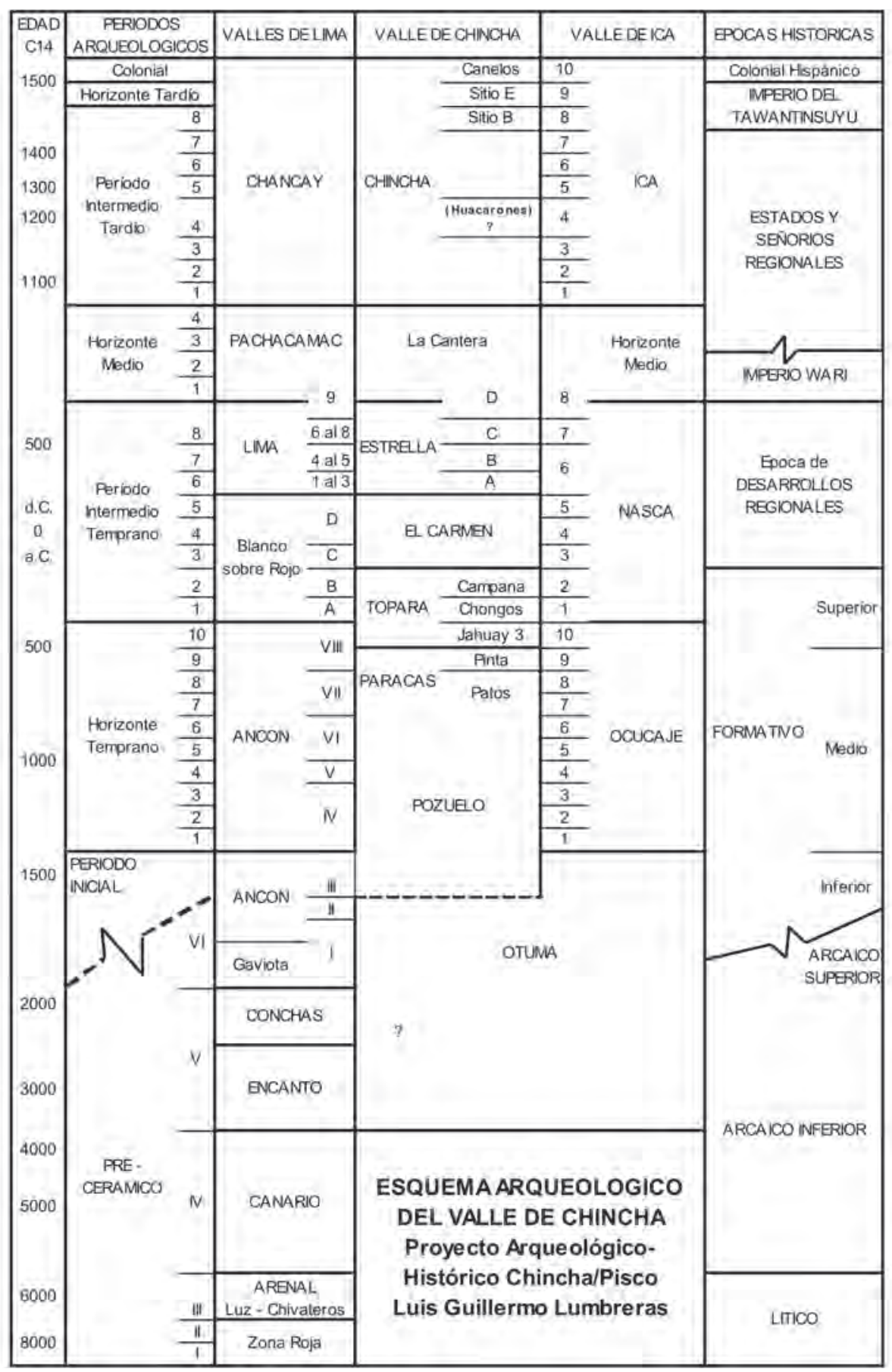

Cuadro 3. Esquema cronológico utilizado por el Programa de Investigaciones Arqueológicas Chincha del INDEA. 
sociedades complejas, la problemática se vuelve más compleja y, por tanto, requiere de otros enfoques adicionales de análisis.

\section{VigENCIA Y PROPUESTAS}

Resulta notable la persistencia de la columna matriz cronológica propuesta por Rowe y sus estudiantes desde los 50, a pesar de los años y todas las investigaciones realizadas en la región, resulta destacable cómo un procedimiento metodológico se ha mantenido vigente y útil. Salvo algunas precisiones producto de los estudios exhaustivos, el método asociativo estilístico aún sirve, creemos, para construir columnas, proponer puntos de partida y plantear derroteros seguros de líneas de investigación y esto es lo que hay que recuperar de Rowe. Él nunca se planteó estandarizar el experimento del sur y repetirlo en toda el área andina; como buen historiador, siempre fue conciente de las particularidades históricas de los grupos sociales y así lo demuestra en todo el cúmulo de estudios historiográficos que produjo sobre su más fascinante tema: la época Inka.

Los estudios que el Proyecto Chincha ha venido desarrollando en los últimos años ha tenido en la propuesta de Rowe un modelo perfectible y valioso que, en este justo homenaje, considero fundamental confesar. El avance de nuestras investigaciones está en la etapa interpretativa y estos inicios de la arqueología del sur, donde predominó el aporte metodológico de John Howland Rowe, constituyen la contribución más palpable de lo que significa ser un verdadero científico social y un riguroso historiador de campo. Rowe es el mejor representante de esta tendencia.

Abundante información se ha producido desde los cincuenta hasta el presente, sobretodo en los últimos diez años, es impostergable la necesidad de incorporar estos datos a la secuencia maestra de Rowe, para seguir perfeccionándola y permitir abrir otras puertas que no sean solamente el estudio cronológico de las sociedades pretéritas. Es necesario incursionar con mayor certeza en los procesos sociales y en las complejidades culturales.

\section{Bibliografía}

Canziani A., José

1990 "Fichas de Reconocimiento del valle de Chincha-1990", Proyecto Chincha del Instituto Andino de Estudios Arqueológicos (manuscrito).

1992a "Patrones de Asentamiento en la Arqueología del valle de Chincha, Perú". En: II Curso de Prehistoria de América Hispana, págs. 87-123. Murcia, Cátedra de Prehistoria de la Universidad de Murcia. Comisión V Centenario de la Región de Murcia y Dirección General de Educación y Universidad de la Comunidad Autónoma de la Región de Murcia.

1992b "Arquitectura y Urbanismo Paracas en el valle de Chincha". En Gaceta Arqueológica Andina № 22: 87-118. INDEA. Lima.

Del Águila Chávez, Carlos

2000a "La Cultura Paracas". En Culturas Prehispánicas. Capítulo 5. Muxica Editores. Diario El Comercio, Universidad Particular Ricardo Palma. Lima.

2000b "La Cultura Chincha". En Culturas Prehispánicas. Capítulo 21. Muxica Editores. Diario El Comercio, Universidad Particular Ricardo Palma. Lima.

2002 "Plateros precoloniales tardíos en Tambo de Mora, valle de Chincha (Siglos XIV-XVI)". En ANALES 10 Museo de América. Conjuntamente con Javier Alcalde; Fernando Fujita y Enrique Retamozo. Pp.: 43-56. Madrid, España.

Instituto Andino de Estudios Arqueológicos

1996 Investigaciones arqueológicas en el valle de Chincha 1995-1996, Informe Final. Presentado al Instituto Nacional de Cultura y al American Museum of Natural History. Lima. Manuscrito.

1998 Investigaciones arqueológicas en el valle de Chincha 1997, Informe Final. Presentado al Instituto Nacional de Cultura y al American Museum of Natural History. Lima. Manuscrito. 
1999 Investigaciones arqueológicas en el valle de Chincha 1998, Informe Final. Presentado al Instituto Nacional de Cultura y al American Museum of Natural History. Lima. Manuscrito.

Lumbreras S., Luis Guillermo

1884a "Fichas de reconocimiento del valle de Chincha-1984". Proyecto Arqueológico Histórico Chincha-Pisco (manuscrito).

1984b Secuencia cultural poblacional del valle de Chincha. Informe de los trabajos realizados en 1984(manuscrito).

Menzel, Dorothy

1966 "The Pottery of Chincha". En Nawpa Pacha, 4: 77-144 Berkeley.

1976 Pottery style and society in Ancient Peru. Berkeley, University of California Press.

1971 "Estudios arqueológicos en los valles de Ica, Pisco, Chincha y Cañete. Sumario preparado con los resultados de las investigaciones realizadas durante 1957, 1958 y 1959; dentro del Programa Fulbright correspondiente a la costa sur del Perú”. En Arqueología y Sociedad 6. Publicación semestral del Museo de Arqueología y Etnología de la Universidad Nacional Mayor de San Marcos. Lima.

Menzel, Dorothy y John H. Rowe

1966 "The role of Chincha in Late Pre Spanish Perú". En Nawpa Pacha, 4: 63-76. Berkeley.

Morris, Craig

1985 "Proyecto Arqueológico Histórico Chincha-Pisco, Informe General de la Temporada 1984". Department of Anthropology, American Museum of Natural History, New York (manuscrito).
1988 "Más allá de las fronteras de Chincha". En La frontera del Estado Inca, BAR International Series, 442: 131-140.

Rowe, John H.

1958 "La seriación cronológica de la cerámica de Paracas elaborada por Lawrence E. Dawson”. En Revista del Museo Regional de Ica. Año IX, Nº 10, pp. 9-21. Ica.

1959a "Archaeologycal dating and cultural process". Southwestern Journal of Anthropology, Vol. 15, $\mathrm{N}^{\circ} 4$ Winter, 1959. pp. 317-324. Albuquerque.

1959b "Tiempo, estilo y proceso cultural en la arqueología peruana". En: Revista Universitaria, año XLVII, $\mathrm{N}^{\circ} 1152^{\circ}$ semestre de 1958, pp. 79-96, Cusco.

Sandweiss, Daniel

1992 "The Archaeology of Chincha Fishermen: Specialization and Status in Inka Peru". Bulletin of Carnegie Museum of Natural History, 29. Pittsburgh.

Uhle, Max

1924 "Explorations at Chincha". University of California Publications in American Archaeology and Ethnology, 21(1):5794.

Wallace, Dwight

1971 "Sitios arqueológicos del Perú, valles de Chincha y Pisco". En Arqueológicas, 13. Museo Nacional de Antropología y Arqueología, Lima.

1972 "Alcance de la secuencia cultural". Adenda de Arqueológicas 13. Museo Nacional de Antropología y Antropología, Lima. 